\title{
A Fatal Complication of a Peripheral Venous Catheter ${ }^{* \#}$
}

\author{
Lenneke E. M. Haas ${ }^{1}$, Bart C. Kortlandt ${ }^{1}$, Steven F. T. Thijsen ${ }^{2,3}$, Jan-Willem Fijen ${ }^{1}$, \\ Sanjay U. C. Sankatsing ${ }^{4}$ \\ ${ }^{1}$ Department of Intensive Care Medicine, Diakonessenhuis, Utrecht, The Netherlands; ${ }^{2}$ Department of Cardiothoracic Surgery, Uni- \\ versity Medical Center Utrecht, Utrecht, The Netherlands; ${ }^{3}$ Department of Microbiology, Diakonessenhuis, Utrecht, The Netherlands; \\ ${ }^{4}$ Department of Internal Medicine, Diakonessenhuis, Utrecht, The Netherlands. \\ Email: lvlelyveld@diakhuis.nl, Bc.kortlandt@gmail.com, sthijsen@diakhuis.nl,jwfijen@diakhuis.nl, ssankatsing@diakhuis.nl
}

Received May 31 ${ }^{\text {st }}$ 2012; revised June 30 $0^{\text {th }}$ 2012; accepted July $16^{\text {th }}, 2012$

\begin{abstract}
Peripheral venous catheters (PVC) are widely used in the hospital and seem to be innocent. However, complications can be devastating. We present a case of a fatal septic shock due to vertebral osteomyelitis after PVC-related Staphylococcus aureus bacteremia (SAB). Staphylococcus aureus is a leading cause of bacteraemia in both the community and the hospital with a significantly increased incidence over the last several decades. Intravascular catheters are the most common cause of SAB. Morbidity and mortality are high, even with appropriate therapy. Although complications are known and common, they may be difficult to recognize. Vertebral osteomyelitis is one of these known severe complications.
\end{abstract}

Keywords: Staphylococcus Aureus Bacteraemia; Vertebral Osteomyelitis; Peripheral Venous Catheter; Intensive Care

\section{Introduction}

Staphylococcus aureus is a leading cause of bacteraemia and its incidence is still increasing. Predisposing factors of S. aureus bacteraemia (SAB) are older age, intravascular catheters, wounds, needle use, co morbid illnesses (diabetes mellitus, respiratory illness, malignancy) and the use of immunosuppressive drugs [1,2]. Intravascular catheters are the most common cause of SAB [3].

Complications of SAB are common, but may be difficult to recognize. Persistent fever and positive follow-up blood cultures 48 to 96 hours after starting antibiotics are predictive for subsequent complications [4]. Prior to the discovery of antibiotics, SAB was fatal in more than $80 \%$ of cases [5]. Nowadays, its mortality rate is still over $20 \%[6-8]$.

Hematogenous spread is the most frequent cause of osteomyelitis and lumbar vertebral bodies are most often involved [9]. Although the disc space has no direct blood supply, it can become secondary involved [10].

The incidence of vertebral osteomyelitis is about 1:350,000 and has steadily increased during recent years probably as a consequence of the increasing rates of nosocomial bacteraemia due to intravascular devices and other forms of instrumentation, increasing age of the population and more injection drug use [11]. The majority of the patients are older than 50 years of age, whereas

\footnotetext{
*None of the authors has a conflict of interest to declare.

\#All authors contributed to the writing of this case report and they all approved the final version.
}

men are affected approximately twice as often as women [12]. Vertebral osteomyelitis (also named spinal osteomyelitis, spondylodiscitis, septic discitis, or disc space infection) is a known severe complication of Staphylococcus aureus (S. aureus) bacteraemia (SAB) which accounts for more than 50 percent of cases. Other pathogens include Enteric Gram-negative bacilli, Pseudomonas aeruginosa, Candida spp., Groups B and G hemolytic streptococci and Mycobacterium tuberculosis [13].

Pain is the major symptom, fever occurs inconsistently. The majority of patients have an elevated C-reactive protein (CRP) and erythrocyte sedimentation rate (ESR), leucocytes can be elevated or normal [14]. Blood cultures are positive in up to 50 to 70 percent of patients [15]. Standard radiographic imaging often remain normal in the early phases. Computed tomography (CT) can show typical or suggestive changes in an earlier phase, although subtle abnormalities may be missed. Magnetic resonance imaging (MRI) is the most sensitive radiologic technique for vertebral osteomyelitis although, 18F-fluorodeoxyglucose-positron emission tomography (PET) is a new promising tool [16]. The diagnosis can be confirmed by culture of the intervertebral discus or vertebral bone tissue. However, when blood cultures from a patient with compatible clinical and radiographic findings reveal a likely pathogen, a biopsy is not necessary [15].

Antibiotic therapy should be guided by culture results including susceptibility testing. Parenteral antibiotics are recommended [13]. The optimal duration of antibiotic 
treatment is still not completely determined. Although most patients respond to antimicrobial therapy, surgery may be necessary in some patients.

We present a case of a fatal septic shock due to vertebral osteomyelitis after a peripheral venous catheter (PVC)-related SAB. Although SAB is not uncommon, we think this case is informative, because it illustrates that a simple and widely used PVC can have devastating complications, even when adequately treated.

\section{Case Presentation}

A 75-year-old woman was admitted to the orthopedic ward because of severe back pain. Her medical history revealed multiple osteoporotic vertebral fractures. CTimaging of the spine showed an osteoporotic fracture of the 11th thoracic vertebral body (see Figure 1(a)), for which she received oral analgesics and a back bracing. Three days after admission, she collapsed and was transferred to our ICU. Clinical examination showed severe hypotension and a newly developed partial paraplegia. She was resuscitated with fluids and a vasopressor under the suspicion of a neurogenic shock due to spinal compression.

The initial laboratory results are shown in Table $\mathbf{1 .}$

A MRI was performed, demonstrating a considerably decreased and increased signal intensity of both the 10th and 11th thoracic vertebral body and a partial compression of the myelum at this level on respectively $\mathrm{T} 1$ and $\mathrm{T} 2$ weighted images (see Figures 1(b) and (c)).

Subsequently a laminectomy and spondylodesis was performed to relieve the compressed spinal cord.

A review of the patient medical history revealed a PVC-related SAB 3 months before which was treated with intravenous flucloxacillin for two weeks. Because of the suspicion of a pyogenic osteomyelitis, high dose intravenous flucloxacillin (12 gram/day) was started. Gentamicin was initially added, but was stopped after two days. S. aureus was cultured from both blood and from the vertebral disc and paravertebral fatbiopsies. Since at this point an endocarditis was considered a transesophageal echocardiography (TEE) was performed which showed no signs of endocarditis. Peroperatively taken biopsies of the affected vertebral body showed chronic inflammation.

Although initial hemodynamic improvement was seen and signs of partial paraplegia decreased, her clinical condition worsened in the following days. She developed multiple organ dysfunction syndrome (MODS) with hemoynamic, respiratory, renal and intestinal failure and disseminated intravascular coagulation (DIC). Because of the progressive MODS and in view of her poor performance state previously, it was decided to withdraw life sustaining treatment. She deceased soon thereafter.

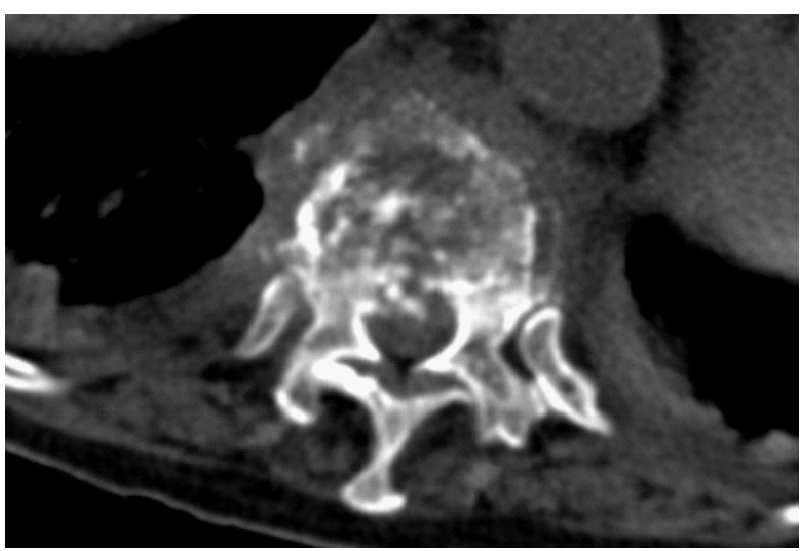

(a)

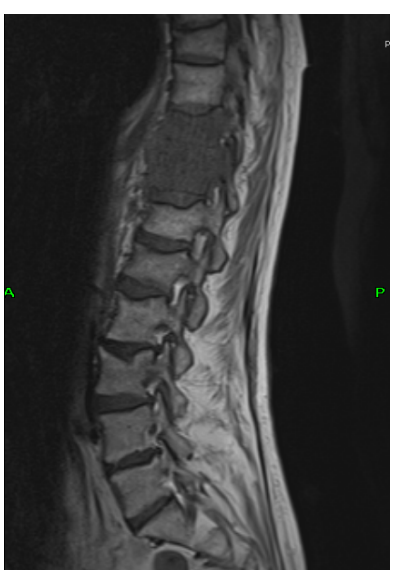

(b)

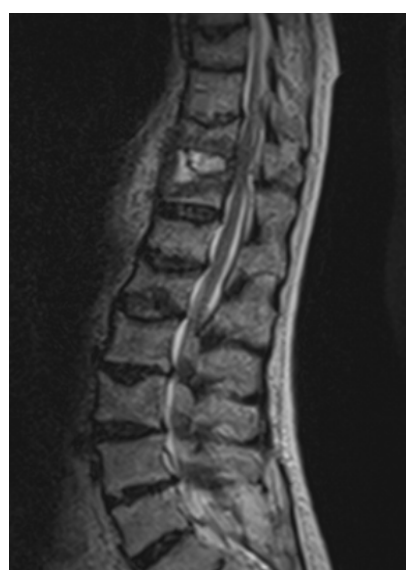

(c)

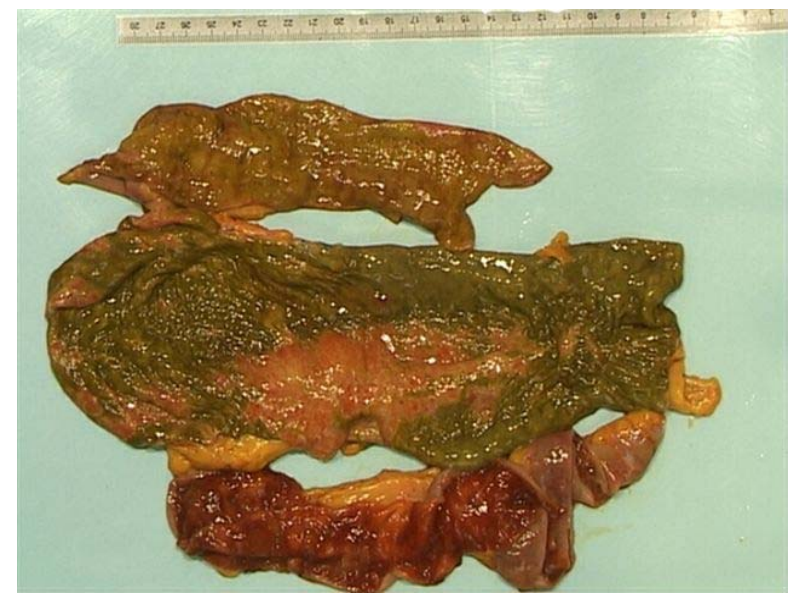

(d)

Figure 1. (a) CT-image of the spine showing a new osteoporotic fracture of the 11th thoracic vertebral body with bone fragments in the vertebral space and a paravertebral hematoma; (b) T1-weighted MRI image, showing low signal intensity at level Th10-Th11, suspected for fracture with edema, metastasis or infection; (c) T2-weighted MRI image, showing a considerably increased signal intensity of both the 10th and 11th thoracic vertebral body and a partial compression of the myelum at this level; (d) Autopsy photograph showing a part of the colon with greenish coating and a large ulceration. 
Table 1. Laboratory results on admission to the ICU.

\begin{tabular}{lcc}
\hline LABORATORY TEST & VALUE & REFERENCE VALUE \\
\hline Leucocytes count & $1.73 \times 10^{10} / \mathrm{L}$ & $4.0-10.0 \times 10^{9} / \mathrm{L}$ \\
C-reactive protein (CRP) & $126 \mathrm{mg} / \mathrm{L}$ & $<10 \mathrm{mg} / \mathrm{L}$ \\
Sodium & $131 \mathrm{mmol} / \mathrm{L}$ & $135-145 \mathrm{mmol} / \mathrm{L}$ \\
Potassium & $4.6 \mathrm{mmol} / \mathrm{L}$ & $3.5-5.0 \mathrm{mmol} / \mathrm{L}$ \\
Urea & $23 \mathrm{mmol} / \mathrm{L}$ & $2.5-6.4 \mathrm{mmol} / \mathrm{L}$ \\
Creatinin & $270 \mu \mathrm{mol} / \mathrm{L}$ & $44-80 \mu \mathrm{mol} / \mathrm{L}$ \\
Gamma-glutamyl transferase (GGT) & $108 \mathrm{U} / \mathrm{L}$ & $<35 \mathrm{U} / \mathrm{LL}$ \\
Alkaline phosphatase (ALP) & $256 \mathrm{U} / \mathrm{L}$ & $35-120 \mathrm{U} / \mathrm{L}$ \\
Alanine aminotransferase (ALT) & $57 \mathrm{U} / \mathrm{L}$ & $<45 \mathrm{U} / \mathrm{L}$ \\
Aspartate aminotransferase (AST) & $130 \mathrm{U} / \mathrm{L}$ & $<40 \mathrm{U} / \mathrm{L}$ \\
Lactase dehydrogenase (LDH) & $397 \mathrm{U} / \mathrm{L}$ & $<220 \mathrm{U} / \mathrm{L}$ \\
Amylase & $616 \mathrm{U} / \mathrm{L}$ & $<100 \mathrm{U} / \mathrm{L}$ \\
Lactate & $10.3 \mathrm{mmol} / \mathrm{L}$ & $0.5-2.2 \mathrm{mmol} / \mathrm{L}$ \\
Albumin & $29 \mathrm{~g} / \mathrm{L}$ & $35-55 \mathrm{~g} / \mathrm{L}$ \\
pH & 7.18 & $7.35-7.45$ \\
$\mathrm{pCO}_{2}$ & $4.7 \mathrm{kPa}$ & $4.7-6.4 \mathrm{kPa}$ \\
$\mathrm{pO}{ }_{2}$ & $17.9 \mathrm{kPa}$ & $10.0-13.3 \mathrm{kPa}$ \\
Bicarbonate & $12.8 \mathrm{mmol} / \mathrm{L}$ & $22-29 \mathrm{mmol} / \mathrm{L}$ \\
\hline
\end{tabular}

Autopsy was performed and revealed an ischemic large intestine with ulcerations and vascular microthrombi (see Figure 1(d)). Ischemia was thought to be due to the combination of DIC with high dose noradrenalin. Further determination of the $S$. aureus was performed and it appeared to be an enterotoxin B producing Panton Valentine Leucocidin (PVL) negative strain which might at least partially explain the pathologic findings of the large intestine. During autopsy no signs of endocarditis were found either.

\section{Discussion}

PVC-related SAB is still an under recognized complication. As presented in our case, this may have catastrophic consequences, even when adequately treated.

What can we learn from this case? In retrospect, the initial treatment might not have been adequate. Since a purulent trombophelitis was present, a complicated SAB should have been suspected and more than two weeks of intravenous therapy should have been considered [17]. Unfortunately, no follow-up blood cultures after initiation of treatment were taken and no TEE was performed. If these cultures were taken and found positive, or in case of development of complications, therapy indeed should have been continued. Adherence to diagnostic and therapeutic guidelines of SAB still seems to be insufficient [18]. It has been demonstrated that consultation of an infectious disease specialist in case of a SAB results in more frequent detection of endocarditis and metastatic infection [19]. In our hospital we have a protocol in which every patient with a SAB is consulted by a resident internal medicine under supervision of an infectious disease specialist or microbiologist. Unfortunately, in this case the protocol was not followed and there had been no consultation.

In addition, there was a critical delay in diagnosis since the complaints of back pain were initially attributed to the known degenerative spinal disease, whereas vertebral osteomyelitis was not considered despite multiple positive blood cultures with S. aureus three months earlier.

\section{Conclusion}

In this patient, a pyogenic vertebral osteomyelitis with catastrophic consequences developed due to a PVC related SAB. This case illustrates that even a simple and widely used item as a PVC can cause serious complications and that in case of a SAB consultation of an infectious disease team might be beneficial. Furthermore, in our opinion, all patients with a SAB, even when a complicated infection is ruled out, should be instructed to contact a physician in case of fever or other signs that might suggest a metastatic infection.

\section{Summary of Key Points}

- Peripheral venous catheters (PVC) are widely used in hospitals and seem very innocent. However complications can be devastating. 
- Staphylococcus aureus is leading cause of bacteraemia in both the community and the hospital and the incidence is still increasing. Intravascular catheters are the most common cause of SAB.

- SAB has a high morbidity and mortality, even with appropriate therapy. Endocarditis and vertebral osteomyelitis are two serious complications of SAB.

- A minimum of two weeks of parenteral antibiotic therapy should be given and follow-up blood cultures should be taken.

- Consultation of an infectious disease specialist seems of great additive value.

\section{Acknowledgements}

The authors would like to thank Dr. L. Wijnaendts, pathologist and L. Sibinga Mulder, radiologist, both from the Diakonessenhuis Utrecht, for their contribution to this case report.

\section{REFERENCES}

[1] G. Jacobsson, S. Dashti, T. Wahlberg and R. Andersson, "The Epidemiology of and Risk Factors for Invasive Staphylococcus Aureus Infections in Western Sweden," Scandinavian Journal of Infectious Diseases, Vol. 39, No. 1, 2007, pp. 6-13. doi:10.1080/00365540600810026

[2] P. Moreillon, Y. A. Que and M. P. Glauser, "Staphylococcus aureus (Including Staphylococcal Toxic Shock)," In: G. L. Mandell, J. E. Bennett and R. Dolin, Eds., Principles and Practice of Infectious Diseases, Churchill Livingstone, Philadelphia, 2005, p. 2321.

[3] A. G. Jensen, C. H. Wachmann, K. B. Poulsen, F. Espersen, J. Scheibel, P. Skinhoj and N. Frimodt-Moller, "Risk Factors for Hospital-Acquired Staphylococcus Aureus Bacteremia,” Archives of Internal Medicine, Vol. 159, No. 13, 1999, pp. 1437-1444.

doi:10.1001/archinte.159.13.1437

[4] R. Khatib, L. B. Johnson, M. G. Fakih, K. Riederer, A. Khosrovaneh, M. Shamse Tabriz, M. Sharma and S. Saeed, "Persistence in Staphylococcus aureus Bacteremia: Incidence, Characteristics of Patients and Outcome," Scandinavian Journal of Infectious Diseases, Vol. 38, No. 1, 2006, pp. 7-14. doi:10.1080/00365540500372846

[5] D. Skinnner and C. S. Keefer, "Significance of Bacteremia Caused by Staphylococcus aureus: A Study of One Hundred and Twenty-Two Cases and a Review of the Literature Concerned with Experimental Infection in Animals," Archives of Internal Medicine, Vol. 68, No. 5, 1941, pp. 851-875. doi:10.1001/archinte.1941.00200110003001

[6] A. F. Shorr, Y. P. Tabak, A. D. Killian, V. Gupta, L. Z. Liu and M. H. Kollef, "Healthcare-Associated Bloodstream Infection: A Distinct Entity? Insights from a Large U.S. Database,” Critical Care Medicine, Vol. 34, No. 10, 2006, pp. 2588-2595. doi:10.1097/01.CCM.0000239121.09533.09

[7] S. Lautenschlager, C. Herzog and W. Zimmerli, "Course and Outcome of Bacteremia due to Staphyloccus aureus: Evaluation of Different Clinical Case Definitions," Clinical Infectious Diseases, Vol. 16, No. 4, 1993, pp. 567573. doi:10.1093/clind/16.4.567

[8] S. J. van Hal, S. O. Jensen, V. L. Vaska, B. A. Espidido, D. L. Paterson and I. B. Gosbell, "Predictors of Mortality in Staphylococcus Aureus Bacteremia,” Clinical Microbiology Reviews, Vol. 25, No. 2, 2012, pp. 362-386. doi:10.1128/CMR.05022-11

[9] W. Zimmerli, "Clinical Practice. Vertebral Osteomyelitis," The New England Journal of Medicine, Vol. 362, No. 11, 2010, pp. 1022-1029. doi:10.1056/NEJMcp0910753

[10] F. J. Vos, C. P. Bleeker-Rovers, P. D. Sturm, P. F. Krabbe, A. P. van Dijk, M. L. Cuijpers, E. M. Adang, G. J. Wanten, B. J. Kullberg and W. J. Oyen, "18F-FDG PET/ CT for Detection of Metastatic Infection in Gram-Positive Bacteremia,” Journal of Nuclear Medicine, Vol. 51, No. 8, 2010, pp. 1234-1240. doi:10.2967/jnumed.109.072371

[11] W. Y. Cheung and K. D. Luk, "Pyogenic Spondylitis," International Orthopaedics, Vol. 36, No. 2, 2012, pp. 397-404. doi:10.1007/s00264-011-1384-6

[12] F. L. Sapico and J. Z. Montgomerie, "Vertebral Osteomyelitis," Infectious Disease Clinics of North America, Vol. 4, 1990, pp. 539-550.

[13] D. P. Lew and F. A. Waldvogel, "Osteomyelitis," The Lancet, Vol. 364, No. 9431, 2004, pp. 369-379. doi:10.1016/S0140-6736(04)16727-5

[14] A. J. Torda, T. Gottlieb and R. Bradbury, "Pyogenic Vertebral Osteomyelitis: Analysis of 20 Cases and Review," Clinical Infectious Diseases, Vol. 20, No. 2, 1995, pp. 320-328. doi:10.1093/clinids/20.2.320

[15] M. J. Patzakis, S. Rao, J. Wilkins, T. M. Moore and P. J. Harvey, "Analysis of 61 Cases Vertebral Osteomyelitis," Clinical Orthopaedics and Related Research, Vol. 264, 1991, pp. 178-183.

[16] S. Ohtori, M. Suzuki, T. Koshi, M. Yamashita, K. Yamauchi, G. Inoue, S. Orita, Y. Eguchi, K. Kuniyoshi, N. Ochiai, S. Kishida, M. Takaso, Y. Aoki, T. Ishikawa, G. Arai, M. Miyagi, H. Kamoda, M. Suzuki, J. Nakamura, T. Toyone and K. Takahashi, “18F-Fluorodeoxyglucose-PET for Patients with Suspected Spondylitis Showing Modic Change,” Spine, Vol. 35, No. 26, 2010, pp. E1599-E1603. doi:10.1097/BRS.0b013e3181d254b4

[17] L. A. Mermel, M. Allon, E. Bouza, D. E. Craven, P. Flynn, N. P. O’Grady, I. I. Raad, B. J. A. Rijnders, R. J. Sherertz and D. K. Warren, "Clinical Practice Guidelines for the Diagnosis and Management of Intravascular Catheter-Related Infection: 2009 Update by the Infectious Diseases Society of America," Clinical Infectious Diseases, Vol. 49, No. 1, 2009, pp. 1-45. doi:10.1086/599376

[18] G. W. D. Landman, J. W. Bouwhuis, P. Bloembergen, J. T. M. van de Meer and P. H. P. Groeneveld, "Evaluation of Diagnosis and Therapy of Staphylococcus Aureus Bacteraemia: Recommended Minimum Treatment Duration of 2 Weeks not Always Met,” Nederlands Tijdschrift voor Geneeskunde, Vol. 155, 2011, pp. 1690-1695.

[19] T. C Jenkins, C. S. Price, A. L. Sabel, P. S. Mehler and W. 
J. Burman, "Impact of Routine Infectious Diseases Service Consultation on the Evaluation, Management, and Outcomes of Staphylococcus Aureus Bacteraemia," Clini- cal Infectious Diseases, Vol. 46, No. 7, 2008, pp. 10001008. doi: $10.1086 / 529190$

\section{Abbreviations}

PVC: Peripheral venous catheter

SAB: Staphylococcus aureus bacteraemia

CRP: C-reactive protein

ESR: Erythrocyte sedimentation rate

CT: Computed tomography
MRI: Magnetic resonance imaging PET: Positron emission tomography TEE: Transesophageal echocardiography MODS: Multiple organ dysfunction syndrome DIC: Disseminatedintravascular coagulation PVL: Panton Valentine Leucocidin 